PROCEEDINGS OF THE

AMERICAN MATHEMATICAL SOCIETY

Volume 138, Number 6, June 2010, Pages 1983-1988

S 0002-9939(10)10244-5

Article electronically published on February 2, 2010

\title{
MONOMIAL REGULAR SEQUENCES
}

\author{
JEFFREY MERMIN
}

(Communicated by Bernd Ulrich)

ABSTRACT. We study Hilbert functions of ideals containing a regular sequence of monomials.

\section{INTRODUCTION}

Let $B=k\left[x_{1}, \ldots, x_{n}\right]$ be a polynomial ring over a field $k$, and let $I \subset B$ be a homogeneous ideal. An important invariant of $I$ is its Hilbert function, $\operatorname{Hilb}_{I}^{B}(d)=$ $\operatorname{dim}_{k} I_{d}$. We study Hilbert functions of ideals containing a regular sequence of monomials.

Macaulay Ma showed in 1927 that all Hilbert functions of homogeneous ideals of $B=k\left[x_{1}, \ldots, x_{n}\right]$ are attained by lex ideals. Over what quotients of $B$ is this true? Let $M$ be a monomial ideal of $B$. We say that $M$ is Lex-Macaulay if every Hilbert function over $B / M$ is attained by a lex ideal of $B / M$. Clements and Lindström CL] proved that $\left(x_{1}^{e_{1}}, \ldots, x_{n}^{e_{n}}\right)$ is Lex-Macaulay for $e_{1} \leq \cdots \leq e_{n}$. In Section 4 we classify the monomial regular sequences which are Lex-Macaulay: Theorem 4.4 says that a regular sequence of monomials is Lex-Macaulay if and only if it has the form $\left(x_{1}^{e_{1}}, \ldots, x_{r-1}^{e_{r-1}}, x_{r}^{e_{r}-1} y\right)$, with $e_{1} \leq \cdots \leq e_{r}$ and $y=x_{i}$ for some $i \geq r$.

The main tool in our proofs is $\mathcal{A}$-compression, which we introduce in Section 3 . It generalizes the notion of $x_{i}$-compression used in CL, MP1, MP2.

\section{Preliminaries}

We give some definitions and notation that are used throughout.

Let $k$ be a field and $B=k\left[x_{1}, \ldots, x_{n}\right]$ be graded by $\operatorname{deg} x_{i}=1$. A monomial $x_{1}^{\alpha_{1}} \ldots x_{n}^{\alpha_{n}}$ has exponent vector $\alpha=\left(\alpha_{1}, \ldots, \alpha_{n}\right)$ and is sometimes written $\mathbf{x}^{\alpha}$. We denote by $|\alpha|$ the degree of $\mathbf{x}^{\alpha},|\alpha|=\sum_{i=1}^{n} \alpha_{i}$.

We order the variables by $x_{1}>\cdots>x_{n}$ and denote by $>_{\text {Lex }}$ the graded lexicographic order on the monomial: $\mathbf{x}^{\alpha}>_{\text {Lex }} \mathbf{x}^{\beta}$ if $|\alpha|>|\beta|$ or if $|\alpha|=|\beta|$ and for some index $j$ we have $\alpha_{j}>\beta_{j}$ and $\alpha_{i}=\beta_{i}$ for all $i<j$. Every monomial $m \neq 1$ has a unique predecessor $n$ in the graded lex order, satisfying $n<_{\text {Lex }} m$ and $n \geq_{\text {Lex }} n^{\prime}$ whenever $m>_{\text {Lex }} n^{\prime}$.

A $d$-vector space is a $k$-vector subspace of $B$ spanned by homogeneous polynomials in degree $d$. A $d$-monomial space is a $d$-vector space spanned by monomials. The dimension of such a space $M_{d}$ will be denoted $\left|M_{d}\right|$. If $I=\left(g_{1}, \ldots, g_{s}\right)$ is a

Received by the editors July 16, 2007, and, in revised form, October 6, 2009.

2010 Mathematics Subject Classification. Primary 13F20.

Key words and phrases. Hilbert function, lexicographic ideals.

(C)2010 American Mathematical Society 
monomial ideal, we denote by $I_{d}$ or $\left(g_{1}, \ldots, g_{s}\right)_{d}$ the degree $d$ component of $I$. Let $\mathbf{m}$ be the monomial space generated by the variables, $\mathbf{m}=\left(x_{1}, \ldots, x_{n}\right)_{1}$. For a $d$-monomial space $M_{d}$, we denote by $\mathbf{m} M_{d}$ the $(d+1)$-monomial space spanned by monomials of the form $x_{i} m$ with $m \in M_{d}$.

A $d$-monomial space $L_{d}$ is lex or a lex segment if whenever $\mathbf{x}^{\alpha}$ and $\mathbf{x}^{\beta}$ are degree $d$ monomials with $\mathbf{x}^{\alpha}$ lexicographically greater than $\mathbf{x}^{\beta}$ and $\mathbf{x}^{\beta} \in L_{d}$, we have $\mathrm{x}^{\alpha} \in L_{d}$ as well. A monomial ideal $L$ is lex if $L_{d}$ is a lex segment for all $d$.

A $d$-monomial space $N_{d}$ is strongly stable or Borel if whenever $i<j$ and $g x_{j} \in N_{d}$ for some monomial $g \in B_{d-1}$, we have $g x_{i} \in N_{d}$ as well. A monomial ideal $N$ is strongly stable or Borel if $N_{d}$ is Borel for all $d$.

A monomial regular sequence is a regular sequence $\left\{f_{1}, \ldots, f_{r}\right\}$ consisting of monomials. Equivalently, it is a collection of monomials satisfying $\operatorname{gcd}\left(f_{i}, f_{j}\right)=1$ for all $i \neq j$.

\section{3. $\mathcal{A}$-COMPRESSiON}

Compressed ideals were used heavily in CL, MP1, MP2]. We introduce $\mathcal{A}$ compressed ideals, which are more general.

Definition 3.1. Let $\mathcal{A}$ be a subset of $\left\{x_{1}, \ldots, x_{n}\right\}$, and let $M_{d}$ be a $d$-monomial vector space. Denote by $\bigoplus_{\mathbf{x}^{\alpha} \in k\left[\mathcal{A}^{c}\right]}$ a direct sum over exponent vectors $\alpha=\left(\alpha_{1}, \ldots, \alpha_{n}\right)$ such that $\alpha_{i}=0$ whenever $x_{i} \in \mathcal{A}$. Then $M_{d}$ may be written uniquely in the form

$$
M_{d}=\bigoplus_{\mathbf{x}^{\alpha} \in k\left[\mathcal{A}^{c}\right]} \mathbf{x}^{\alpha} V_{\alpha}
$$

where $V_{\alpha}$ is a $(d-|\alpha|)$-monomial space in the $\operatorname{ring} k[\mathcal{A}]$. We say that $M_{d}$ is $\mathcal{A}$ compressed if each $V_{\alpha}$ is lex in $k[\mathcal{A}]$.

If $L_{\alpha}$ is the lex monomial space in degree $(d-|\alpha|)$ with $\left|L_{\alpha}\right|=\left|V_{\alpha}\right|$, and $L_{d}=$ $\bigoplus_{\mathbf{x}^{\alpha} \in k\left[\mathcal{A}^{c}\right]} \mathbf{x}^{\alpha} L_{\alpha}$, we say that $L_{d}$ is the $\mathcal{A}$-compression of $M_{d}$.

Remark. What [L, MP1, MP2 called $i$-compressed ideals (or monomial spaces) are $\left\{x_{1}, \ldots, \widehat{x_{i}}, \ldots, x_{n}\right\}$-compressed in this notation. (Here the $\widehat{x_{i}}$ means that $x_{i}$ is omitted.) This reversal is for simplicity in several proofs below and so that $\mathcal{A}$ compressed spaces will remain $\mathcal{A}$-compressed after new variables are added to the ring $B$.

Example 3.2. If $\mathcal{A}$ is a one-element set, every monomial space is $\mathcal{A}$-compressed.

Example 3.3. Let $M_{3}=\left\langle a^{3}, a^{2} b, a^{2} c, a b^{2}, a b c, a b d, b^{3}, b^{2} c\right\rangle$. Then $M_{3}$ is not $\{a, b\}$ compressed because $V_{d}=\langle a b\rangle$ is not lex in $k[a, b]$, but $M_{3}$ is $\mathcal{A}$-compressed for every other two-element set $\mathcal{A}$.

Lemma 3.4. Let $M_{d}$ be $\mathcal{A}$-compressed. Then $\mathbf{m} M_{d}$ is $\mathcal{A}$-compressed as well.

Definition 3.5. A monomial ideal $M$ is $\mathcal{A}$-compressed if it is $\mathcal{A}$-compressed in every degree $d$.

It is easy to prove the following two propositions:

Proposition 3.6. A lex ideal (or lex monomial space) is $\mathcal{A}$-compressed for every $\mathcal{A}$. 
Proposition 3.7. Suppose that $\mathcal{A} \supset \mathcal{B}$. Then every $\mathcal{A}$-compressed ideal (or $\mathcal{A}$ compressed monomial space) is $\mathcal{B}$-compressed.

Example 3.8. Borel ideals are $\mathcal{B}$-compressed for every two-element set $\mathcal{B}$.

Due to the following lemma, $\mathcal{A}$-compressed ideals are useful in the study of Hilbert functions:

Lemma 3.9. Let $M_{d}$ be a d-monomial vector space, and let $K_{d}$ be its $\mathcal{A}$-compression. Then $\left|\mathbf{m} K_{d}\right| \leq\left|\mathbf{m} M_{d}\right|$.

Proof. Let $\mathbf{n}$ be the monomial space generated by the variables of $\mathcal{A}$. Denote by $\beta \uparrow \alpha$ the property that $\mathbf{x}^{\beta}$ divides $\mathbf{x}^{\alpha}$ and $\operatorname{deg} \mathbf{x}^{\beta}=\operatorname{deg} \mathbf{x}^{\alpha}-1$.

Write $M_{d}=\bigoplus_{\mathbf{x}^{\alpha} \in k\left[\mathcal{A}^{c}\right]} \mathrm{x}^{\alpha} N_{\alpha}$ and $K_{d}=\bigoplus_{\mathbf{x}^{\alpha} \in k\left[\mathcal{A}^{c}\right]} \mathrm{x}^{\alpha} L_{\alpha}$. Now we have $\mathbf{m} M_{d}=$ $\bigoplus_{\mathbf{x}^{\alpha} \in k\left[\mathcal{A}^{c}\right]} \mathrm{x}^{\alpha} N_{\alpha}^{\prime}$ and $\mathbf{m} K_{d}=\bigoplus_{\mathbf{x}^{\alpha} \in k\left[\mathcal{A}^{c}\right]} \mathrm{x}^{\alpha} L_{\alpha}^{\prime}$, where

$$
\begin{aligned}
& N_{\alpha}^{\prime}=\mathbf{n} N_{\alpha}+\sum_{\beta \uparrow \alpha} N_{\beta}, \\
& L_{\alpha}^{\prime}=\mathbf{n} L_{\alpha}+\sum_{\beta \uparrow \alpha} L_{\beta} .
\end{aligned}
$$

Since $\left|\mathbf{n} L_{\alpha}\right| \leq\left|\mathbf{n} N_{\alpha}\right|$ by Macaulay's Theorem and $\left|N_{\beta}\right|=\left|L_{\beta}\right|$ for all $\beta$ by definition, we have

$$
\left|L_{\alpha}^{\prime}\right|=\max \left\{\max _{\beta \uparrow \alpha}\left\{\left|L_{\beta}\right|\right\},\left|\mathbf{n} L_{\alpha}\right|\right\} \leq \max \left\{\max _{\beta \uparrow \alpha}\left\{\left|N_{\beta}\right|\right\},\left|\mathbf{n} N_{\alpha}\right|\right\} \leq\left|N_{\alpha}^{\prime}\right|,
$$

the first equality because the $L_{\beta}$ and $\mathbf{n} L_{\alpha}$ are lex segments, so their sum is the longest segment. This proves the lemma.

Example 3.10. We use compression to prove the monomial case of the EisenbudGreen-Harris Conjecture. Eisenbud, Green, and Harris EGH1, EGH2 made the following conjecture motivated by applications in algebraic geometry:

Conjecture 3.11 (Eisenbud-Green-Harris). Let $N$ be any homogeneous ideal containing a regular sequence in degrees $e_{1} \leq \cdots \leq e_{r}$. There is a lex ideal $L$ such that $N$ and $L+\left(x_{1}^{e_{1}}, \ldots, x_{r}^{e_{r}}\right)$ have the same Hilbert function.

Using Lemma 3.9 we prove Conjecture 3.11 for ideals containing a regular sequence of monomials. This special case has been known in folklore for some time but, as near as I can tell, has never been formally written up. (In the folklore proof, the compression is replaced by a coordinate change and an initial ideal.)

Proposition 3.12. Let $R=\left\{f_{1}, \ldots, f_{r}\right\}$ be a regular sequence of monomials with $\operatorname{deg} f_{i}=e_{i}$ and $e_{1} \leq \cdots \leq e_{r}$. Let $N$ be any homogeneous ideal containing $R$. Then there is a lex ideal $L$ such that $N$ and $L+\left(x_{1}^{e_{1}}, \ldots, x_{r}^{e_{r}}\right)$ have the same Hilbert function.

Proof. Set $P=\left(x_{1}^{e_{1}}, \ldots, x_{r}^{e_{r}}\right)$. It suffices to show that, for any $d$-vector space $N_{d}$ containing $(R)_{d}$, there is a lex monomial space $L_{d}$ such that $\left|L_{d}+P_{d}\right|=\left|N_{d}\right|$ and $\left|\mathbf{m} L_{d}+P_{d+1}\right| \leq\left|\mathbf{m} N_{d}\right|$.

By renaming the variables if necessary, we may assume without loss of generality that $x_{i}$ divides $f_{i}$ for all $i$. By Gröbner basis theory, we may assume (after taking an initial ideal if necessary) that $N_{d}$ is a monomial space. Set $N(0)=N_{d}$. For each 
$i \leq r$, let $\mathcal{A}_{i}$ be the set of variables dividing $f_{i}$, and let $N(i)$ be the $\mathcal{A}_{i}$-compression of $N(i-1)$.

We claim that whenever $d \geq e_{j}, N(i)$ contains $x_{j}^{e_{j}}$ if $j \leq i$, and contains $f_{j}$ if $j>i$. Clearly, this statement is true when $i=0$; we prove it by induction on $i$. Suppose $d \geq e_{j}$. If $i \neq j$, the monomial in question is contained in $N(i-1)$ by

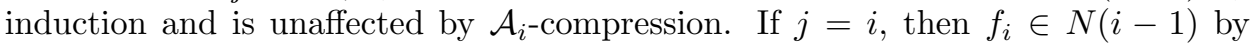
induction, so $x_{i}^{e_{i}} \in N(i)$ as desired.

Furthermore, $|N(i)|=|N(i-1)|$ and $|\mathbf{m} N(i)| \leq|\mathbf{m} N(i-1)|$ by Lemma 3.9. In particular, $N(r)$ contains $P_{d}$ and satisfies $|N(r)|=|N|$ and $|\mathbf{m} N(r)| \leq|\mathbf{m} N|$. By Clements-Lindström's theorem, there is a lex space $L_{d}$ such that $\left|L_{d}+P_{d}\right|=|N(r)|$ and $\left|\mathbf{m} L_{d}+P_{d+1}\right| \leq|\mathbf{m} N(r)|$.

\section{Lex-Macaulay monomial Regular Sequences}

Throughout this section, let $M$ be a monomial ideal of $B$. We recall the following definitions and results from MP1]:

Definition 4.1 ([MP1]). An ideal $M$ is Lex-Macaulay if every Hilbert function in the quotient $B / M$ is attained by a lex ideal. Equivalently, $M$ is Lex-Macaulay if for every $d$ and every $d$-monomial vector space $V_{d}$, there exists a lex space $L_{d}$ such that $\left|L_{d}+M_{d}\right|=\left|V_{d}+M_{d}\right|$ and $\left|\mathbf{m} L_{d}+M_{d+1}\right| \leq\left|\mathbf{m} V_{d}+M_{d+1}\right|$.

Theorem 4.2 ([MP1]). If $M$ is Lex-Macaulay as an ideal of $B$, then $M B[y]$ is Lex-Macaulay as an ideal of $B[y]$.

Proposition 4.3 (MP1]). If $M$ is Lex-Macaulay and $L$ is lex, then $L+M$ is Lex-Macaulay.

We characterize the monomial regular sequences which are Lex-Macaulay as follows.

Theorem 4.4. Let $R$ be the ideal generated by a regular sequence of monomials. Then $R$ is Lex-Macaulay if and only if $R=\left(x_{1}^{e_{1}}, \ldots, x_{r-1}^{e_{r-1}}, x_{r}^{e_{r}-1} y\right)$, with $e_{1} \leq \cdots \leq$ $e_{r}$ and $y=x_{i}$ for some $i \geq r$.

The necessary condition is given by Lemma 4.6, and the sufficient condition by Lemmas 4.7 and 4.8 below.

Remark. The sufficient condition of Theorem 4.4 is a special case of the following more general result, which was suggested by the referee. The proof is the same, modulo the necessary changes in notation. (In the statement of Proposition 4.5, $R$ is a polarization of $Q$; see for example [Fa.)

Proposition 4.5. Suppose that $Q$ is a Lex-Macaulay monomial ideal, and let $x_{r}$ be the least variable dividing a minimal monomial generator of $Q$. Write $Q=U+x_{r} V$, where $x_{r}$ does not divide any minimal generator of $U$. Then $R=U+x_{r+1} V$ is Lex-Macaulay as well.

Lemma 4.6. Let $R$ be the ideal of a monomial regular sequence $\left\{f_{1}, \ldots, f_{r}\right\}$, with the $f_{i}$ ordered so that $i<j$ if $\operatorname{deg} f_{i}<\operatorname{deg} f_{j}$ or if $\operatorname{deg} f_{i}=\operatorname{deg} f_{j}$ and $f_{i}>_{\text {Lex }} f_{j}$. Suppose that $R$ is Lex-Macaulay. Then:

(i) If $i \neq r$, then $f_{i}=x_{i}^{e_{i}}$.

(ii) $x_{r}^{e_{r}-1}$ divides $f_{r}$. 
Proof. We use induction on $i$. Suppose first that $i=1$. If $g$ is any monomial of degree $e_{1}-1$ dividing $f_{1}$, we must have $\left|\left(x_{1}^{e_{1}-1}+R\right)_{e_{1}}\right| \leq\left|(g+R)_{e_{1}}\right| \leq\left|R_{e_{1}}+n-1\right|$; i.e., $x_{1}^{e_{1}-1}$ must divide $f_{1}$. If $r \neq 1$, put $q=e_{1}+e_{2}-1$ and consider $g=x_{1}^{e_{1}-1} h$, where $h$ is any monomial of degree $e_{2}-1$ dividing $f_{2}$. We have $\left|(g+R)_{q}\right|=$ $\left|R_{q}\right|+n-2$. If $f_{1} \neq x_{1}^{e_{1}}$, we would also have $\left|\left(x_{1}^{q-1}+R\right)\right|_{q}=\left|R_{q}\right|+n-1$, contradicting the assumption that $R$ is Lex-Macaulay.

In general, $R+\left(x_{1}, \ldots, x_{i-1}\right)$ is Lex-Macaulay by Proposition 4.3. By induction, $R+\left(x_{1}, \ldots, x_{i-1}\right)=\left(x_{1}, \ldots, x_{i-1}, f_{i}, \ldots, f_{r}\right)$, so $\left(f_{i}, \ldots, f_{r}\right)$ is Lex-Macaulay as an ideal of $k\left[x_{i}, \ldots, x_{r}\right]$. The lemma follows from the $i=1$ case above.

Lemma 4.7. Suppose $r \neq n$, and let $R=\left(x_{1}^{e_{1}}, \ldots, x_{r-1}^{e_{r-1}}, x_{r}^{e_{r}-1} x_{n}\right)$. Let $N_{d}$ be a dmonomial vector space containing $R_{d}$. Then there exists a d-monomial vector space $K_{d}$ containing $Q_{d}$, where $Q=\left(x_{1}^{e_{1}}, \ldots, x_{r-1}^{e_{r-1}}, x_{r}^{e_{r}-1} x_{n-1}\right)$ such that $\left|K_{d}\right|=\left|M_{d}\right|$ and $\left|\mathbf{m} K_{d}\right| \leq\left|\mathbf{m} M_{d}\right|$.

Proof. Set $\mathcal{A}=\left\{x_{n-1}, x_{n}\right\}$, and take $K_{d}$ to be the $\mathcal{A}$-compression of $M_{d}$. Apply Lemma 3.9] and note that $Q$ is the $\mathcal{A}$-compression of $R$.

Lemma 4.8. Suppose $r \neq n$, and let $R=\left(x_{1}^{e_{1}}, \ldots, x_{r-1}^{e_{r-1}}, x_{r}^{e_{r}-1} x_{n}\right)$ and $Q=$ $\left(x_{1}^{e_{1}}, \ldots, x_{r-1}^{e_{r-1}}, x_{r}^{e_{r}-1} x_{n-1}\right)$. Let $L_{d}$ be a lex segment in $B$. Then there exists a lex segment $T_{d}$ such that $\left|T_{d}+R_{d}\right|=\left|L_{d}+Q_{d}\right|$ and $\left|\mathbf{m} T_{d}+R_{d+1}\right|=\left|\mathbf{m} L_{d}+Q_{d+1}\right|$.

Proof. Let $T_{d}$ be the smallest lex segment such that $T_{d}+Q_{d}=L_{d}+Q_{d}$.

We claim that $T_{d}$ satisfies the following property: If $g$ is a monomial such that $g x_{r}^{e_{r}-1} x_{n-1} \in T_{d}$, then $g x_{r}^{e_{r}-1} x_{n} \in T_{d}$. We will prove this claim. Observe that $g x_{r}^{e_{r}-1} x_{n-1} \in Q_{d}$, and that $g x_{r}^{e_{r}-1} x_{n}$ is the predecessor of $g x_{r}^{e_{r}-1} x_{n-1}$ in the graded lex order. By construction, if $g x_{r}^{e_{r}-1} x_{n-1} \in T_{d}$, we must have a monomial $v \in T_{d}$ such that $v<_{\text {Lex }} g x_{r}^{e_{r}-1} x_{n-1}$ and $v \notin Q_{d}$. Then $v \leq_{\text {Lex }} g x_{r}^{e_{r}-1} x_{n}$ as well, and since $T_{d}$ is a lex segment, we have $g x_{r}^{e_{r}-1} x_{n} \in T_{d}$, proving the claim.

Set $A=T_{d}+\left(x_{1}^{e_{1}}, \ldots, x_{r-1}^{e_{r-1}}\right)_{d}$ and let $C$ and $D$ be the monomial spaces such that $T_{d}+Q_{d}=A \oplus C$ and $T_{d}+R_{d}=A \oplus D$. Then if $\Gamma$ and $\Delta$ are the sets of monomials of $C$ and $D$, respectively, we have

$$
\begin{aligned}
\Gamma & =\left\{g x_{r}^{e_{r}-1} x_{n-1} \in B_{d}: g x_{r}^{e_{r}-1} x_{n-1} \notin A\right\}, \\
\Delta & =\left\{g x_{r}^{e_{r}-1} x_{n} \in B_{d}: g x_{r}^{e_{r}-1} x_{n} \notin A\right\} \\
& =\left\{g x_{r}^{e_{r}-1} x_{n} \in B_{d}: g x_{r}^{e_{r}-1} x_{n-1} \notin A\right\} \\
& =\left\{g x_{r}^{e_{r}-1} x_{n} \in B_{d}: g x_{r}^{e_{r}-1} x_{n-1} \in C\right\},
\end{aligned}
$$

the penultimate equality following from the claim above.

In particular, multiplication by $\frac{x_{n}}{x_{n-1}}$ is a bijection from $\Gamma$ to $\Delta$. Thus $|\mathbf{m} C|=$ $|\mathbf{m} D|$ and (again applying the claim) $|\mathbf{m} C \cap \mathbf{m} A|=|\mathbf{m} D \cap \mathbf{m} A|$, so we have $\left|\mathbf{m}\left(T_{d}+R_{d}\right)\right|=\left|\mathbf{m}\left(T_{d}+Q_{d}\right)\right|=\left|\mathbf{m}\left(L_{d}+Q_{d}\right)\right|$, the first equality by inclusionexclusion, the second by construction.

Now $\left|\mathbf{m} T_{d}+R_{d+1}\right|=\left|\mathbf{m}\left(T_{d}+R_{d}\right)\right|$ unless $R$ has minimal monomial generators in degree $d+1$ which are not in $\mathbf{m} T_{d}$; likewise $\left|\mathbf{m} L_{d}+Q_{d+1}\right|=\left|\mathbf{m}\left(L_{d}+Q_{d}\right)\right|$ unless $Q$ has minimal monomial generators in degree $d+1$ which are not in $\mathbf{m} L_{d}$ and hence not in $\mathbf{m} T_{d}$. Since $x_{r}^{d} x_{n-1} \in \mathbf{m} T_{d}$ if and only if $x_{r}^{d} x_{n} \in \mathbf{m} T_{d}$, we obtain $\left|\mathbf{m} T_{d}+R_{d+1}\right|=\left|\mathbf{m} L_{d}+Q_{d+1}\right|$, as desired.

The preceding lemmas combine to prove Theorem 4.4 , 
Proof of Theorem 4.4. If $R$ is Lex-Macaulay, apply Lemma 4.6 .

Conversely, suppose $R=\left(x_{1}^{e_{1}}, \ldots, x_{r-1}^{e_{r-1}}, x_{r}^{e_{r}-1} y\right)$ as in the statement of the theorem. By Theorem 4.2. we may assume $y=x_{n}$. If $n=r$, this is ClementsLindström's theorem; otherwise, we induct on $n-r$. Set $Q=\left(x_{1}^{e_{1}}, \ldots, x_{r-1}^{e_{r-1}}\right.$, $\left.x_{r}^{e_{r}-1} x_{n-1}\right)$. Choose a degree $d$, and let $N_{d}$ be any $d$-monomial space containing $R_{d}$. By Lemma 4.7, there is a $d$-monomial space $K_{d}$ containing $Q_{d}$ with $\left|K_{d}\right|=\left|N_{d}\right|$ and $\left|\mathbf{m} K_{d}\right| \leq\left|\mathbf{m} N_{d}\right| . Q$ is Lex-Macaulay by induction, so there is a lex monomial space $L_{d}$ containing $Q_{d}$ with $\left|L_{d}\right|=\left|K_{d}\right|$ and $\left|\mathbf{m} L_{d}\right| \leq\left|\mathbf{m} K_{d}\right|$. By Lemma 4.8 there is a lex monomial space $T_{d}$ containing $R_{d}$ with $\left|T_{d}\right|=\left|L_{d}\right|$ and $\left|\mathbf{m} T_{d}\right|=\left|\mathbf{m} L_{d}\right|$. Thus $R$ is Lex-Macaulay.

\section{ACKNOWLEDGEMENTS}

The author thanks David Eisenbud for asking which monomial regular sequences are Lex-Macaulay. He thanks Irena Peeva, Steve Sinnott, and Mike Stillman for helpful discussions. The author is particularly grateful to Irena Peeva for considerable help in editing this paper. Finally, he is grateful to the referee for many suggestions improving the precision and clarity of the final paper.

\section{REFERENCES}

[CL] G. Clements and B. Lindström, A generalization of a combinatorial theorem of Macaulay, J. Combinatorial Theory 7 (1969), 230-238. MR0246781 (40:50)

[EGH1] D. Eisenbud, M. Green, and J. Harris, Higher Castelnuovo theory, Astérisque 218 (1993), 187-202. MR.1265314 (95a:14057)

[EGH2] — Cayley-Bacharach theorems and conjectures, Bull. Amer. Math. Soc. 33 (1996), 295-324. MR.1376653 (97a:14059)

[Fa] S. Faridi, Monomial ideals via square-free monomial ideals, Commutative algebra, Lect. Notes Pure Appl. Math., vol. 244, Chapman \& Hall/CRC, Boca Raton, FL, 2006. MR2184792(2006i:13038)

[Ma] F. Macaulay, Some properties of enumeration in the theory of modular systems, Proc. London Math. Soc. 26 (1927), 531-555.

[MP1] J. Mermin and I. Peeva, Lexifying ideals, Math. Res. Lett. 13 (2006), 409-422. MR.2231127 (2007b:13023)

[MP2] _ Hilbert functions and lex ideals, J. Algebra 313 (2007), 642-656. MR2329561 (2008c:13019) 74078

Department of Mathematics, Oklahoma State University, Stillwater, Oklahoma 